\title{
Mapping the Side-Chain Length of Small-Molecule Acceptors towards the Optimal Hierarchical Morphology in Ternary Organic Solar Cells
}

\author{
Zichun Zhou ${ }^{a, b}$ \\ Shengjie $X^{* a}$ \\ Xiaozhang Zhu*a,b (D) \\ ${ }^{a}$ Beijing National Laboratory for Molecular Sciences, CAS Key Laboratory of Organic \\ Solids, Institute of Chemistry, Chinese Academy of Sciences, Beijing 100190, P. R. \\ China \\ ${ }^{\mathrm{b}}$ School of Chemical Sciences, University of Chinese Academy of Sciences, Beijing \\ 100049, P. R. China \\ xushengjie@iccas.ac.cn; xzzhu@iccas.ac.cn
}

Dedicated to Professor Peter Bäuerle on the occasion of his 65th birthday.
Received: 17.01.2021

Accepted after revision: 18.03 .2021

DOI: 10.1055/a-1472-7302; Art ID: om-21-0012sc

License terms: (c)

(c) 2021. The Author(s). This is an open access article published by Thieme under the terms of the Creative Commons Attribution-NonDerivative-NonCommercial License, permitting copying and reproduction so long as the original work is given appropriate credit. Contents may not be used for commercial purposes, or adapted, remixed, transformed or built upon. (https://creativecommons.org/licenses/by-nc-nd/4.0/)

Abstract Using multiple light-absorbing materials to realize a broader and better absorption spectrum in multi-component organic photovoltaics has achieved significant success to obtain high power conversion efficiency. Meanwhile, the good materials combinations with matched electronic structure and proper blend morphology for charge generation and transport are of primary importance for implementation of the multi-component strategy. Hierarchical morphology has been clearly demonstrated to improve all performance parameters in ternary organic photovoltaics but shows strong dependence on the molecular structures. Here we develop four small-molecule electron acceptors with different alkyl chain lengths to find the optimal solution of alkyl chain towards the defined hierarchical morphology and carry out a clear and comprehensive investigation of the alkyl chain length effects on the structure-morphology-device performance relationships in ternary blends. There is a positive correlation between the power conversion efficiencies of the four ternary systems and their short-circuit current density parameters, manifesting the significance of distinguishing optimal alkyl side chain length of small-molecule electron acceptors for defined hierarchical morphology to afford efficient carrier generation. The non-optimal side chains would retard the BTR crystallization and make the $\mathrm{PC}_{71} \mathrm{BM}$ domain sizes incontrollable, leading to a morphology without a defined hierarchy. Such a detailed mapping of the alkyl side chain length of small-molecule electron acceptors provides new insight into the materials combinations for the next-step high-performance multicomponent organic photovoltaics.

Key words ternary organic solar cells, hierarchical morphology, sidechain modulation, small-molecule acceptors

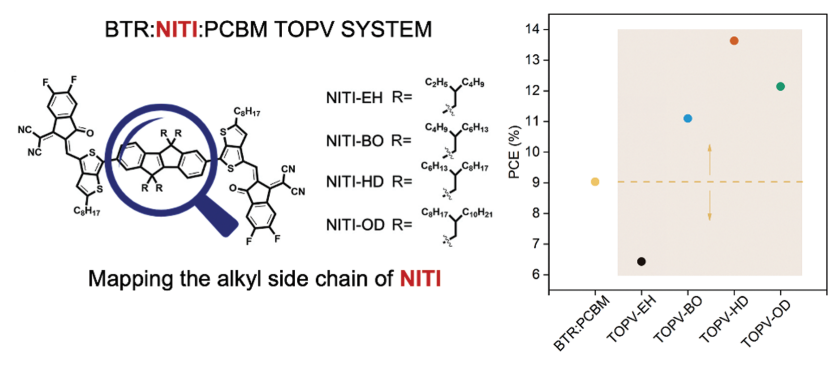

\section{Introduction}

Multi-component organic photovoltaics (OPVs) comprising multiple light-absorbing materials to realize a broader and better absorption spectrum have achieved a significant success to obtain high power conversion efficiency (PCE). ${ }^{1-7}$ The materials combinations with matched electronic structures and proper blend morphology for charge generation and transport can help suppress the energy loss and improve the thickness tolerability, indicating a vital role of the multicomponent strategy for the commercial success of the OPV technique.,9 By resorting to ternary (TOPV) or quaternary (QOPV) blend system, recent research studies have led to a rapid progress in all-small-molecule OPVs with PCEs approaching $16 \%$ and polymer-based OPVs with PCEs over $17 \%{ }^{10-17}$ The development of multi-component OPVs strongly relies on a trial-and-error approach among various materials, which needs a lot of repeated attempts. In consideration of the crystallization, aggregation, and surface-tension properties of different materials, manipulating the morphology of multicomponent blends to balance the carrier generation and transport and reduce the energy or current loss channels is very complicated and challenging. In our recent work, we put forward the "donor-acceptor-fullerene" (BTR:NITI:PC ${ }_{71} \mathrm{BM}$ ) ternary hierarchical morphology concept, where $\mathrm{PC}_{71} \mathrm{BM}$ framework affords charge transport highways and intricate BTR:NITI small-sized phase separation is in charge of carrier generation and the control of the energy losses. ${ }^{9}$ This success was benefited from the thorough understanding of material properties, and we successfully applied unfavorable interactions between small donor/acceptor molecules and the fullerene acceptor to frame a large-sized network, and then optimize the detailed inter-network phase separation and interfacial orientations. It is for the first time that a "hierarchical morphology" has been clearly demonstrated to improve all parameter space in organic solar cells, and the 
morphology, photophysical function as well as the device performance have been quite well correlated. We are delighted to see that some latest works share the similar morphologycontrol methodology and even obtained an outstanding QOPV performance with PCEs over 18\% recorded in the Best ResearchCell Efficiency Chart from NERL (National Renewable Energy Laboratory). ${ }^{18}$ In the meantime, we found that NITI-EH with a short side chain (2-ethylhexyl) has a profound effect in controlling the morphology of the ternary blends leading to a morphology without a defined hierarchy. Given that the alkyl chain length of small-molecule non-fullerene acceptors plays an important role in manipulation of solubility, crystalline behavior, and miscibility in the blends, it is prevalent to investigate the detailed alkyl chain length effects in binary OPVs but less concerned in TOPV systems. ${ }^{13,19-24}$

The NITI-HD has shown considerable success in the BTR: NITI:PC ${ }_{71}$ BM ternary strategy with an outstanding PCE of 13.6\%. ${ }^{9}$ Here we further collect four NITI electron acceptors with different alkyl chain lengths named NITI-EH to NITI-OD to find the optimal solution of alkyl chain length towards the defined hierarchical morphology by carrying out a clear and comprehensive investigation of the alkyl-chain-length effect on the structure-morphology-device performance relationship. The difference in alkyl chain length does not cause noteworthy changes in electronic structure but changes the molecular stacking behaviors in the respective solid film to a great extent. When blended in ternary systems (BTR:NITI:PC ${ }_{71} \mathrm{BM}$ ), the NITIEH-based TOPV blend suffers heavy losses in structure order, such as weakness in $\pi-\pi$ stacking order and oversized $\mathrm{PC}_{71} \mathrm{BM}$ framework. In contrast, the NITI-HD-based TOPV blend shows a quite similar structure order to the $\mathrm{BTR}: \mathrm{PC}_{71} \mathrm{BM}$ binary blend without retarding BTR crystallization. NITI-BO- and NITI-ODbased TOPV blends both show a moderate molecular packing feature, but more efficiently than the NITI-EH-based TOPV blend. The structural features of these ternary blends strongly reflect the status of the small-sized BTR:NITI-rich regions, which determines the efficiency of carrier generation. Consequently, the NITI-EH-based TOPV exhibits a much lower PCE of $6.43 \%$ than the binary host BTR:PC ${ }_{71} \mathrm{BM}$ system (9.03\%). The NITI-HD-based TOPV shows a remarkable improvement in PCE with a champion PCE of 13.63\%. The NITI-BO- and NITI-ODbased TOPVs both show moderate increases in PCE with values of $11.10 \%$ and $12.14 \%$, respectively. Moreover, there is a positive correlation between the PCEs of the four TOPV systems and their short-circuit current density $\left(J_{s c}\right)$, manifesting the significance of distinguishing optimal alkyl side chain length of NITI for defined hierarchical morphology to afford efficient carrier generation and charge transport.

\section{Results and Discussion}

The synthesis and molecular characterization of the new molecules NITI-BO and NITI-OD are shown in
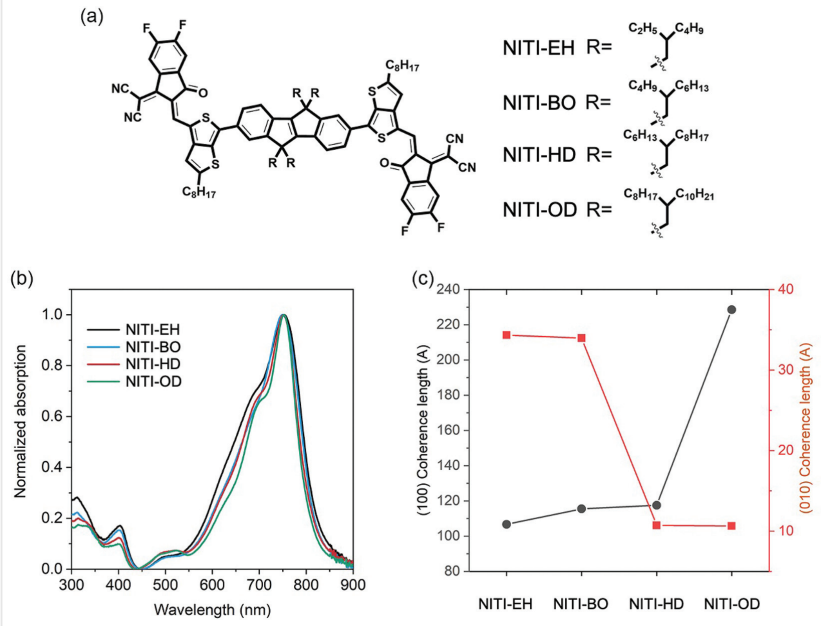

Figure 1 a) Chemical structures of the NITI acceptor family possessing alkyl side chain with different lengths. b) Normalized UV-vis-NIR absorption spectra of the four NITI small-molecule acceptors in thin film. c) crystallinity coherence lengths of the four NITI small-molecule acceptors measured by grazing incidence $\mathrm{X}$-ray diffraction.

the Supporting Information (SI). Figure 1(a, b) shows the chemical structures and absorption profiles of the four NITI electron acceptors used in this study. The maximum absorption peaks and optical bandgaps of all four acceptors are nearly identical in the thin films. The HOMO and LUMO energy levels measured by ultraviolet photoelectron spectroscopy coupled with the optical band gaps also show similar results (Figure S1, see SI). The molecular stacking behaviors of the four NITI electron acceptors shown Figure S2 (see SI) were measured by grazing incidence X-ray diffraction (GIXD), and the corresponding length information of the crystallinity coherence is shown in Figure 1c. The NITI-EH shows a (100) reflection in the inplane (IP) direction at $0.36 \AA^{-1}$ and a quite sharp $\pi-\pi$ diffraction peak in the out-of-plane (OOP) direction at $1.84 \AA^{-1}$, showing crystal coherence lengths of 10.6 and $3.4 \mathrm{~nm}$, respectively, estimated by the Scherrer equation. ${ }^{25}$ NITI-BO displays a quite similar structure ordering to NITIEH. However, for NITI-HD and NITI-OD, much suppressed $\pi-$ $\pi$ stacking peak is seen, which is mainly due to the existence of the long bended alkyl side chain on the conjugated backbone to form steric hindrance, influencing the electrostatic interaction in the $\pi-\pi$ direction, thus the crystallization is inhibited. In addition, the (100) diffraction peaks both can be found in IP and OOP directions for NITI-HD and NITI-OD, indicating different molecular packing behaviors compared to those of NITI-EH and NITI-BO. Pole figure analysis was further performed, as shown in Figure S3 (see SI), with missing wedge labeled. ${ }^{26}$ For NITI-EH and NITI-BO thin films, the (100) peak is rich in the horizontal direction, while the $\pi-\pi$ stacking peak mainly locates in the vertical direction, indicating a face-on orientation. But for the NITI- 
(a)

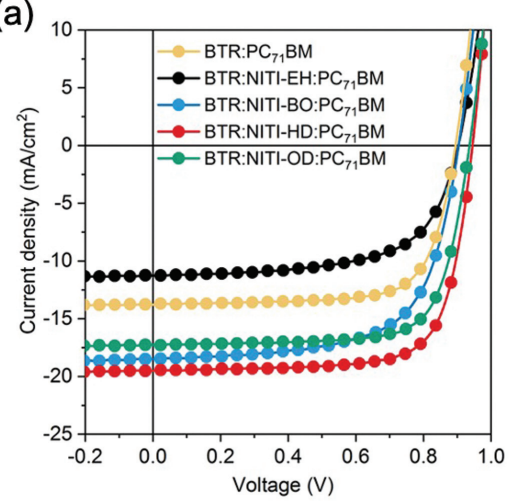

(b)

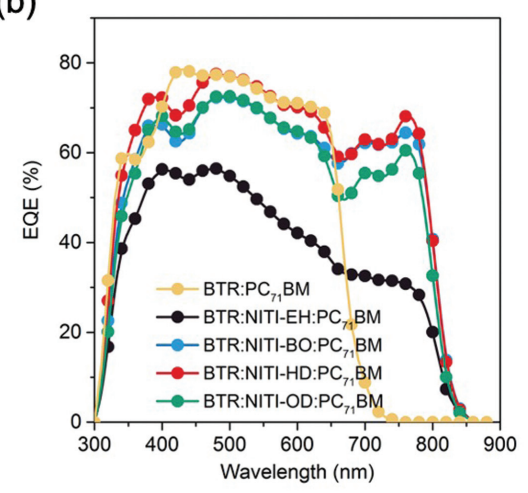

(c)

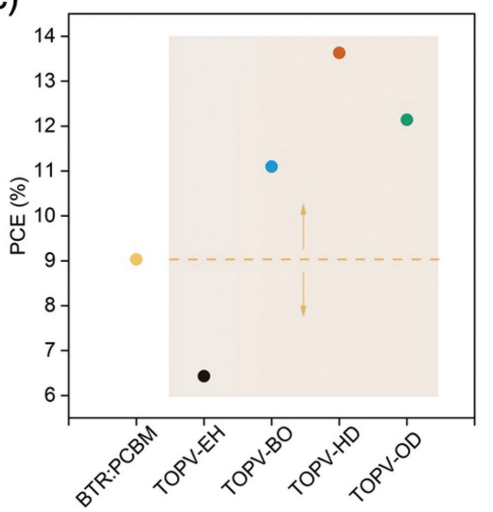

Figure 2 a) Current-voltage characteristics and b) EQE curves for the four ternary devices under AM $1.5 \mathrm{G}$ irradiation (100 $\left.\mathrm{mW} \mathrm{cm}^{-2}\right)$.c) visualization of PCEs' comparison.

HD and NITI-OD thin films, both (100) and $\pi-\pi$ stacking peak locate in the vertical direction, indicating the existence of both face-on and edge-on orientations.

TOPV devices were fabricated with a conventional configuration and the active layer was spin-coated from a chloroform solution of BTR, NITI and PC71 BM with a fixed weight ratio of 1:0.4:1. The post-treatment of tetrahydrofuran (THF) solvent vapor annealing (SVA) was used during morphology optimization. Figure 2a shows the current density-voltage $(J-V)$ curves and the key device parameters are summarized in Table 1. Device based on the BTR:NITI$\mathrm{EH}: \mathrm{PC}{ }_{71} \mathrm{BM}$ ternary (TOPV-EH) blend shows a reduced PCE of $6.43 \%$ with an open-circuit voltage $\left(V_{\text {oc }}\right)$ of $0.90 \mathrm{~V}$, a $J_{\text {sc }}$ of $11.26 \mathrm{~mA} \mathrm{~cm}^{-2}$, and a fill factor (FF) of $63.49 \%$. The poor device performance indicates the failure of forming a defined hierarchical morphology. In contrast, device based on the BTR:NITI-BO:PC ${ }_{71} \mathrm{BM}$ ternary (TOPV-BO) blend shows an enhanced PCE of $11.10 \%$ with a $V_{\text {oc }}$ of $0.92 \mathrm{~V}$, a $J_{\text {sc }}$ of $18.49 \mathrm{~mA} \mathrm{~cm}^{-2}$, and a $\mathrm{FF}$ of $66.43 \%$. Further, device based on the BTR:NITI-HD:PC ${ }_{71}$ BM ternary (TOPV-HD) blend shows a champion PCE of $13.63 \%$ with a $V_{\text {oc }}$ of $0.94 \mathrm{~V}, \mathrm{a} J_{\mathrm{sc}}$ of $19.50 \mathrm{~mA} \mathrm{~cm}^{-2}$, and a FF of $73.83 \%$. When the NITI-OD was added in the ternary system, a TOPV-OD device shows a moderate PCE of $12.14 \%$ with a $V_{\mathrm{oc}}$ of $0.94 \mathrm{~V}$, a $J_{\mathrm{sc}}$ of $17.35 \mathrm{~mA} \mathrm{~cm}^{-2}$, and a FF of $74.09 \%$. The external quantum

Table 1 Summary of photovoltaic parameters of the four ternary devices under AM $1.5 \mathrm{G}$ irradiation $\left(100 \mathrm{~mW} \mathrm{~cm}^{-2}\right)$

\begin{tabular}{lllll}
\hline Blends & $V_{\text {oc }}(\mathrm{V})$ & $J_{\text {sc }}\left(\mathrm{mA} \mathrm{cm}^{-2}\right)$ & Fill factor $(\%)$ & PCE (\%) \\
\hline TOPV-EH & 0.90 & 11.26 & 63.49 & 6.43 \\
TOPV-BO & 0.92 & 18.49 & 66.43 & 11.10 \\
TOPV-HD & 0.94 & 19.50 & 73.83 & 13.63 \\
TOPV-OD & 0.94 & 17.35 & 74.09 & 12.14 \\
\hline
\end{tabular}

efficiency (EQE) curves of the four TOPV devices are shown in Figure 2b. Only the EQE of the TOPV-HD blend is as high as that of the BTR:PC ${ }_{71} \mathrm{BM}$ binary blend in the range of 300-650 nm. The TOPV-EH blend shows poor EQEs in the whole absorption spectrum. TOPV-BO and TOPV-OD blends show reduced EQEs compared to the TOPV-HD blend in the range of $300-650 \mathrm{~nm}$ and $300-800 \mathrm{~nm}$, respectively. The integrated $J_{\mathrm{sc}}$ values from the EQE spectra for the four TOPV devices match well with the $J_{\text {sc }}$ values from the $J-V$ curves within a $3 \%$ error. We further measured the mobilities of the faster carrier components via photo-induced charger-carrier extraction in linearly increasing voltage (photo-CELIV) measurements. ${ }^{27-29}$ Unlike the spacecharge-limited-currents (SCLC) model using single carrier device configuration to measure charge mobilities, the photo-CELIV method can do better in analyzing the charge transport by using the photovoltaic devices directly. The same photovoltaic device configuration used in the photo-CELIV method and $J-V$ characterization can avoid interference factor from device interface differences, which may further result in changes in blend films. The mobilities calculated from the curves are summarized in Figure 3b. The
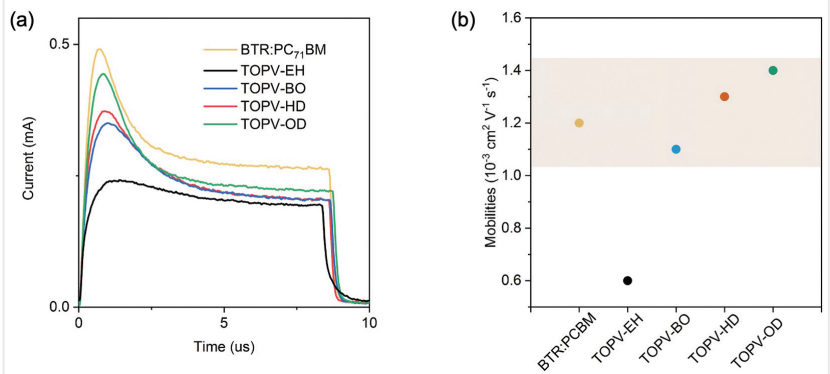

Figure 3 a) Mobilities measured by photo-induced charge carrier extraction in linearly increasing voltage. b) The mobility values obtained from the curves. 
(a)

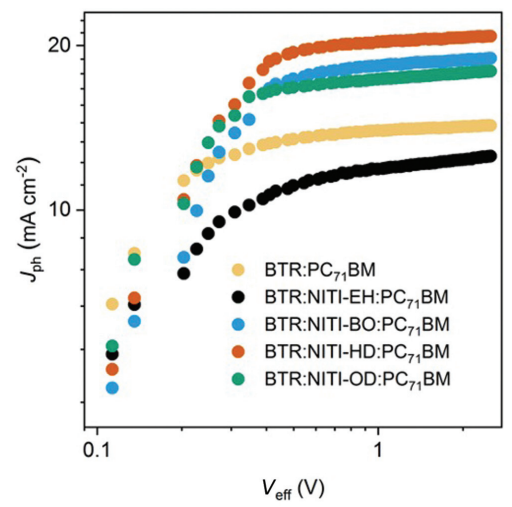

(b)

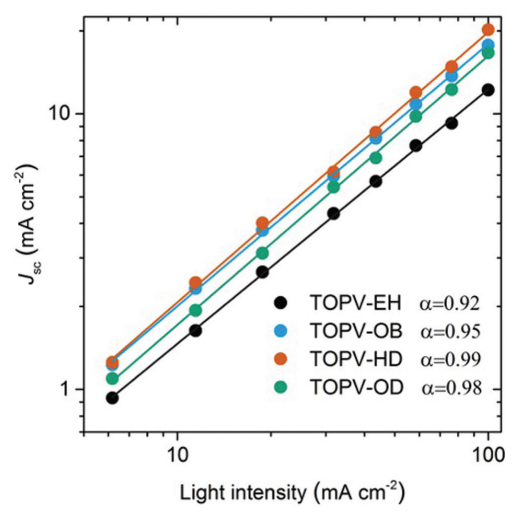

(c)

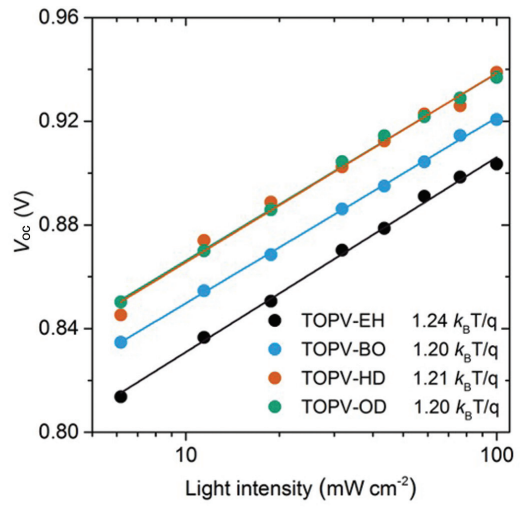

Figure 4 a) Characteristics of the photocurrent density versus effective voltage $\left(U_{\mathrm{ph}}-V_{\text {eff }}\right)$. b) Measurement of $J_{\mathrm{sc}}$ versus light intensity. c) $V_{\mathrm{oc}}$ versus light intensity for ternary devices.

TOPV-EH blend shows the worst mobility indicating the absence of a well-organized morphology framework. The mobilities of the remaining three TOPV blends exhibit similar values around $1 \times 10^{-3} \mathrm{~cm}^{2} \mathrm{~V}^{-1} \mathrm{~s}^{-1}$, being consistent with the performance improvement in these three devices than the BTR:PC ${ }_{71}$ BM binary device.

Charge generation was examined by analyzing the dependence of photocurrent density $\left(J_{\mathrm{ph}}\right)$ on the effective voltage $\left(V_{\mathrm{eff}}\right) \cdot J_{\mathrm{ph}}$ is defined as $J_{\mathrm{ph}}=J_{\mathrm{L}}-J_{\mathrm{D}}$, where $J_{\mathrm{L}}$ and $J_{\mathrm{D}}$ represent the current densities that are illuminated and in the dark, respectively. $V_{\text {eff }}$ is defined as $V_{0}-V_{\mathrm{a}}$, where $V_{0}$ represents the voltage when $J_{\mathrm{ph}}$ is 0 and $V_{\mathrm{a}}$ represents the applied voltage bias. ${ }^{23,30-32}$ As shown in Figure $4 \mathrm{a}$, the $J_{\mathrm{ph}}$ values of both the binary and ternary devices increase to its saturation value $\left(J_{\text {sat }}\right)$, and the $J_{\text {sat }}$ values are in direct proportion to the $J_{s c}$ values, testifying that differences in charge generation determine the $J_{\text {sc }}$ values and then PCE values. In addition, the $J_{\mathrm{ph}} / J_{\mathrm{sat}}$ ratio is also used to determine the overall efficiencies of charge collections vs. charge recombination. The TOPV-HD and TOPV-OD blends show similar values of $97 \%$ and $86 \%$, respectively, under shortcircuit and maximum output power conditions, respectively, leading to improved $J_{\mathrm{sc}}$ and high FF values. However, the TOPV-EH and TOPV-BO blends show quite low values of 76\% and $77 \%$, respectively, under the maximum output power conditions, indicating insufficient charge transport and collection. The free carriers suffer from nongeminate recombination if they cannot be extracted and transported to the electrodes. Nongeminate recombination can be dominated by trap-assisted (monomolecular) or bimolecular mechanisms. ${ }^{33-35}$ The recombination mechanism was studied by measuring the dependencies of $J_{\mathrm{sc}}$ and $V_{\mathrm{oc}}$ on light intensity. ${ }^{30}$ The relationship between $V_{\mathrm{oc}}$ and light intensity can be used to distinguish whether trap-assisted recombination (slope of $2 k_{\mathrm{B}} T / q$, where $k_{\mathrm{B}}$ is Boltzmann's constant and $T$ represents the absolute temperature) is the dominant mechanism at open circuit. As exhibited in Figure 4(b, c), TOPV-EH shows a larger slope than those of the remaining three devices, indicating the more trapassisted recombination in TOPV-EH. In order to further determine bimolecular recombination in these four ternary devices, we investigated the variation of $J_{\mathrm{sc}}$ as a function of light intensity at short circuit. The best fit for the data is obtained when the value $\alpha$ is close to unity, indicating negligible bimolecular recombination in a system. The TOPV-EH device shows a slope $(\alpha)$ of 0.92 , which is the lowest value in this study. Thus, the TOPV-EH device suffers from considerable bimolecular recombination losses. The TOPV-BO device also exhibit a low slope $(\alpha)$ of 0.95 , leading to the poor FF value. In contrast, the TOPV-HD- and TOPVOD-based devices both show neglectable bimolecular recombination losses.

Further, GIXD and resonant soft X-ray scattering (RSoXS) were measured to offer insight into the influence of the morphology features on the charge generation. ${ }^{36-38}$ Figure 5 $(\mathrm{a}-\mathrm{c})$ shows the line-cut profiles of GIXD diffraction patterns and crystallinity analysis. For the TOPV-EH blend, the crystallization of BTR is retarded and the diffraction patterns are dominated by strong crystallization of NITI-EH electron acceptor. As a result, the TOPV-EH blend suffers heavy losses in structure order, such as weakness in $\pi-\pi$ stacking order. The current ternary blends are processed via spin-coating, followed by THF SVA, as such an SVA treatment is crucial during morphology optimization and performance enhancement. The high crystallinity BTR donor is expected to crystallize when the BHJ thin film is vitrified via THF SVA. Thus, the low structure order of the TOPV-EH blend suggests the unfavorable morphology features of the small-sized BTR:NITI-rich regions to afford sufficient charge generation. When performing the RSoXS experiment, the X-ray energy is tuned near the atomic absorption edge of constituent elements, for example the $\mathrm{K}$ absorption edge of carbon at 
(a)

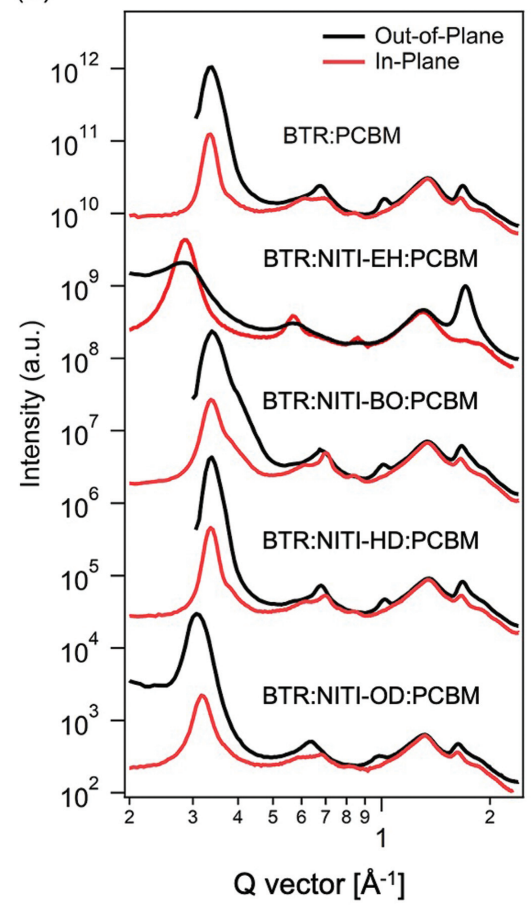

(b)

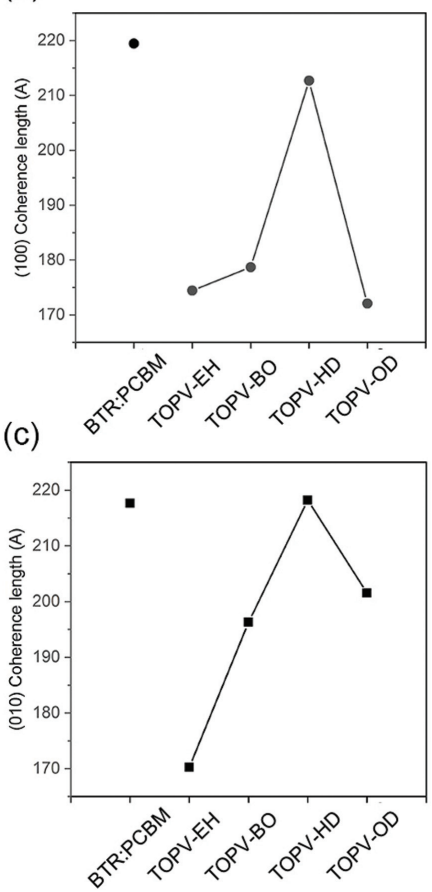

(d)

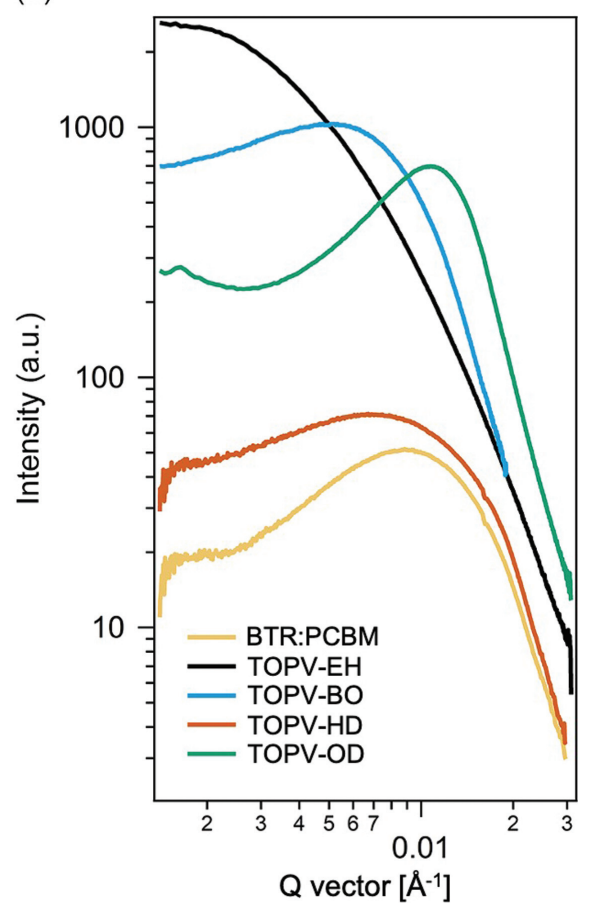

Figure 5 a) In-plane and out-of-plane line-cut profiles of the two-dimensional GIXD data. b) The estimated (100) crystal size of the blend films. c) The estimated (010) crystal size of the blend films. d) RSoXS scattering profiles of the binary and ternary thin films.

$284.2 \mathrm{eV}$, where $\mathrm{PC}_{71} \mathrm{BM}$ shows much better response, to provide enough chemical sensitivity. It is more rigorous to claim that the contrast of characteristic absorption between the $\mathrm{PC}_{71} \mathrm{BM}$-rich phase and the BTR:NITI phase at this X-ray energy highlights the information of $\mathrm{PC}_{71} \mathrm{BM}$ domains. In our previous work, NITI-HD could mix well with BTR, with interfacial preference to confirm efficient exciton dissociation. ${ }^{9}$ Thus, the original contrast in BTR:PCBM maintains. The other three NITI could aggregate to form its own phase, showing a totally different contrast compared with the BTR: $\mathrm{PC}_{71} \mathrm{BM}$ thin film, thus, a much different scattering shape is seen. The enhanced scattering intensity may be the result of improved phase purity. ${ }^{39}$ In detail, the phase-separation size of the TOPV-EH blend measured by RSoXS is too large to be estimated. The larger and purer $\mathrm{PC}_{71} \mathrm{BM}$-rich domains would further damage the efficiency of charge transfer from BTR:NITI-rich regions to the $\mathrm{PC}_{71} \mathrm{BM}$ domains and result in considerable charge recombination. TOPV-BO and TOPV-OD blends both show moderate molecular packing features in GIXD results and overlarge scattering intensity in RSoXS results. The TOPV-BO blend also shows a large phaseseparation size of the $\mathrm{PC}_{71} \mathrm{BM}$ framework over $100 \mathrm{~nm}$, leading to the insufficient $J_{\mathrm{sc}}$ and FF values. What makes the TOPV-HD blend special is that it shows quite similar structure order in GIXD result and phase-separation scattering shape to the BTR:PC ${ }_{71} \mathrm{BM}$ binary blend. The small increase in phase-separation size and scattering intensity compared with the BTR:PC ${ }_{71} \mathrm{BM}$ binary blend results from the swell of $\mathrm{PC}_{71} \mathrm{BM}$ domains when NITI-HD loading increases. Overall, the favorable structure order and defined $\mathrm{PC}_{71} \mathrm{BM}$ framework in TOPV-HD blend realize the high efficiencies of charge generation and transport.

\section{Conclusions}

We fully investigate the side-chain length of NITI electron acceptor in a ternary system to gain insight into the alkyl chain length effects on the structure-morphology-device performance relationships for the optimal hierarchical morphology. The alkyl side chain length has a profound impact on the ternary morphology features and determines the final photovoltaic performance. Only the NITI-HD-based ternary blend shows a defined large-scale $\mathrm{PC}_{71} \mathrm{BM}$ framework to afford charge transport and sufficient structure order in the small-scale BTR:NITI regions, where charge generation performs. The short or too long side chains would retard the BTR crystallization and make the $\mathrm{PC}_{71} \mathrm{BM}$ domain sizes incontrollable. Such a detailed mapping of the alkyl side chain length of small-molecule electron acceptors provides an insightful view into the optimization of materials combinations for the next-step high-performance TOPV blends. 


\section{Funding Information}

This work was financially supported by the National Key R\&D Program of China (2017YFA0204700), the Strategic Priority Research Program of the Chinese Academy of Sciences (XDB12010200), the National Natural Science Foundation of China (21572234, 21661132006, 91833304, 21805289) and the Youth Innovation Promotion Association CAS (No. 2020031).

\section{Supporting Information}

Supporting Information for this article is available online at https://doi.org/10.1055/a-1472-7302.

\section{References and Notes}

Fabrication of organic solar cells: The devices were developed with a conventional structure of ITO/PEDOT:PSS/active layer/PNDIT-F3N/Al. The ITO-coated glass substrates were cleaned with sequential ultrasonication in a soap-deionized water mixture, deionized water, acetone and isopropanol. The washed substrates were further treated with oxygen plasma for 10 min to eliminate any remaining organic components. A thin layer (approximately $30 \mathrm{~nm}$ ) of PEDOT:PSS (Clevios P VP 4083) was first spin-coated on the ITO substrates at 3,000 r.p.m. and baked at $150{ }^{\circ} \mathrm{C}$ for $5 \mathrm{~min}$ under ambient conditions. The substrates were then transferred into a nitrogen-filled glove box. Subsequently, the active layer was spin-coated on the PEDOT: PSS layer via spin-coating from a chloroform solution of BTR, NITI and $\mathrm{PC}_{71} \mathrm{BM}$ at various spin rates. The best PCE was achieved via spin coating $25 \mathrm{mg}$ BTR, $10 \mathrm{mg}$ NITI and $25 \mathrm{mg}$ $\mathrm{PC}_{71} \mathrm{BM}$ (1:0.4:1) in $1 \mathrm{ml}$ chloroform at 1,500 r.p.m. The resultant film thickness was approximately $300 \mathrm{~nm}$ and obtained via a surface profilometer (Dektak XT, Bruker). Here, SVA with THF was used to optimize the blend morphology and promote device performance. SVA was conducted in a $60-\mathrm{mm}$ glass Petri dish containing $200 \mu \mathrm{L}$ THF at various durations in a glove box. The optimal duration in this study was $60 \mathrm{~s}$. Then, PNDIT-F3N (as the electron transport layer) was spin-coated on the active layer at 3,000 r.p.m. from the alcohol solution. In the final stage, aluminium (100 nm) was thermally evaporated onto the active layer as the top electrode. Shadow masks were used to define the device area $\left(0.03262 \mathrm{~cm}^{2}\right)$ of the devices..

Device characterization: The current density-voltage $(J-V)$ characteristics of unencapsulated photovoltaic devices were measured under $\mathrm{N}_{2}$ using a Keithley 2400 source meter. A $300 \mathrm{~W}$ xenon arc solar simulator (Oriel) calibrated by a standard $\mathrm{Si}$ diode was used to simulate the AM $1.5 \mathrm{G}$ solar irradiation. The EQE was performed using certified IPCE equipment..

(1) Gasparini, N.; Salleo, A.; McCulloch, I.; Baran, D. Nat. Rev. Mater. 2019, 4, 229.

(2) Lu, L.; Kelly, M. A.; You, W.; Yu, L. Nat. Photonics 2015, 9, 491.

(3) Xu, X.; Li, Y.; Peng, Q. Nano Select 2020, 1, 30.

(4) Naveed, H. B.; Ma, W. Joule 2018, 2, 621.

(5) Huang, W.; Cheng, P.; Yang, Y. M.; Li, G.; Yang, Y. Adv. Mater. 2018, 20, 1705706.
(6) Baran, D.; Ashraf, R. S.; Hanifi, D. A.; Abdelsamie, M.; Gasparini, N.; Röhr, J. A.; Holliday, S.; Wadsworth, A.; Lockett, S.; Neophytou, M.; Emmott, C. J.; Nelson, J.; Brabec, C. J.; Amassian, A.; Salleo, A.; Kirchartz, T.; Durrant, J. R.; McCulloch, I. Nat. Mater. 2017, 16, 363.

(7) Gasparini, N.; Jiao, X.; Heumueller, T.; Baran, D.; Matt, G. J.; Fladischer, S.; Spiecker, E.; Ade, H.; Brabec, C. J.; Ameri, T. Nat. Energy 2016, 1, 16118.

(8) Zhang, J.; Zhao, Y.; Fang, J.; Yuan, L.; Xia, B.; Wang, G.; Wang, Z.; Zhang, Y.; Ma, W.; Yan, W.; Su, W.; Wei, Z. Small 2017, 2, 1700229.

(9) Zhou, Z.; Xu, S.; Song, J.; Jin, Y.; Yue, Q.; Qian, Y.; Liu, F.; Zhang, F.; Zhu, X. Nat. Energy 2018, 3, 952.

(10) Nian, L.; Kan, Y.; Gao, K.; Zhang, M.; Li, N.; Zhou, G.; Jo, S. B.; Shi, X.; Lin, F.; Rong, Q.; Liu, F.; Zhou, G.; Jen, A. K.-Y. Joule 2020, 4, 2223.

(11) Song, J.; Li, C.; Zhu, L.; Guo, J.; Xu, J.; Zhang, X.; Weng, K.; Zhang, K.; Min, J.; Hao, X.; Zhang, Y.; Liu, F.; Sun, Y. Adv. Mater. 2019, 31 1905645.

(12) Zhan, L.; Li, S.; Lau, T.-K.; Cui, Y.; Lu, X.; Shi, M.; Li, C.-Z.; Li, H.; Hou, J.; Chen, H. Energy Environ. Sci. 2020, 13, 635.

(13) Jiang, K.; Wei, Q.; Lai, J. Y. L.; Peng, Z.; Kim, H. K.; Yuan, J.; Ye, L.; Ade, H.; Zou, Y.; Yan, H. Joule 2019, 3, 3020.

(14) Arunagiri, L.; Peng, Z.; Zou, X.; Yu, H.; Zhang, G.; Wang, Z.; Lai, J. Y. L.; Zhang, J.; Zheng, Y.; Cui, C.; Huang, F.; Zou, Y.; Wong, K. S.; Chow, P. C. Y.; Ade, H.; Yan, H. Joule 2020, 4, 1790.

(15) Xiao, Z.; Jia, X.; Ding, L. Sci. Bull. 2017, 62, 1562.

(16) Qin, J.; Zhang, L.; Xiao, Z.; Chen, S.; Sun, K.; Zang, Z.; Yi, C.; Yuan, Y.; Jin, Z.; Hao, F.; Chen, Y.; Bao, Q.; Ding, L. Sci. Bull. 2020, 65, 1979.

(17) Liu, L.; Liu, Q.; Xiao, Z.; Yang, S.; Yuan, Y.; Ding, L. Sci. Bull. 2019, 64, 1083.

(18) Zhang, M.; Zhu, L.; Zhou, G.; Hao, T.; Qiu, C.; Zhao, Z.; Hu, Q.; Larson, B. W.; Zhu, H.; Ma, Z.; Tang, Z.; Feng, W.; Zhang, Y.; Russell, T. P.; Liu, F. Nat. Commun. 2021, 12, 309.

(19) Zhou, Z.; Liu, W.; Zhou, G.; Zhang, M.; Qian, D.; Zhang, J.; Chen, S.; Xu, S.; Yang, C.; Gao, F.; Zhu, H.; Liu, F.; Zhu, X. Adv. Mater. 2019, 32, 1906324.

(20) Pang, S.; Zhang, R.; Duan, C.; Zhang, S.; Gu, X.; Liu, X.; Huang, F.; Cao, Y. Adv. Energy Mater. 2019, 9, 1901740.

(21) Cui, Y.; Yao, H.; Zhang, J.; Xian, K.; Zhang, T.; Hong, L.; Wang, Y.; Xu, Y.; Ma, K.; An, C.; He, C.; Wei, Z.; Gao, F.; Hou, J. Adv. Mater. 2020, 32, 1908205.

(22) Gao, K.; Miao, J.; Xiao, L.; Deng, W.; Kan, Y.; Liang, T.; Wang, C.; Huang, F.; Peng, J.; Cao, Y.; Liu, F.; Russell, T. P.; Wu, H.; Peng, X. Adv. Mater. 2016, 28, 4727.

(23) Hong, L.; Yao, H.; Wu, Z.; Cui, Y.; Zhang, T.; Xu, Y.; Yu, R.; Liao, Q.; Gao, B.; Xian, K.; Woo, H. Y.; Ge, Z.; Hou, J. Adv. Mater. 2019, 31, 1903441.

(24) Liu, T.; Pan, X.; Meng, X.; Liu, Y.; Wei, D.; Ma, W.; Huo, L.; Sun, X.; Lee, T. H.; Huang, M.; Choi, H.; Kim, J. Y.; Choy, W. C.; Sun, Y. Adv. Mater. 2017, 29, 1604251.

(25) Song, J.; Zhang, M.; Yuan, M.; Qian, Y.; Sun, Y.; Liu, F. Small Methods 2018, 2, 1700229.

(26) Jiang, Z. J. Appl. Crystallogr. 2015, 48, 917.

(27) Min, J.; Luponosov, Y. N.; Gasparini, N.; Richter, M.; Bakirov, A. V.; Shcherbina, M. A.; Chvalun, S. N.; Grodd, L.; Grigorian, S.; Ameri, T.; Ponomarenko, S. A.; Brabec, C. J. Adv. Energy Mater. 2015, 5, 1500386.

(28) Bange, S.; Schubert, M.; Neher, D. Phys. Rev. B: Condens. Matter 2010, 81, 035209. 
(29) Juska, G.; Arlauskas, K.; Viliunas, M.; Kocka, J. Phys. Rev. Lett. 2000, 84, 4946.

(30) Kyaw, A. K. K.; Wang, D. H.; Luo, C.; Cao, Y.; Nguyen, T.-Q.; Bazan, G. C.; Heeger, A. J. Adv. Energy Mater. 2014, 4, 1301469.

(31) Zhang, G.; Xia, R.; Chen, Z.; Xiao, J.; Zhao, X.; Liu, S.; Yip, H.-L.; Cao, Y. Adv. Energy Mater. 2018, 8, 1801609.

(32) Lenes, M.; Morana, M.; Brabec, C. J.; Blom, P. W. M. Adv. Funct. Mater. 2009, 19, 1106.

(33) Proctor, C. M.; Kuik, M.; Nguyen, T.-Q. Prog. Polym. Sci. 2013, 38, 1941.

(34) Cowan, S. R.; Roy, A.; Heeger, A. J. Phys. Rev. B: Condens. Matter 2010, 82, 245207.
(35) Ran, N. A.; Love, J. A.; Heiber, M. C.; Jiao, X.; Hughes, M. P.; Karki, A.; Wang, M.; Brus, V. V.; Wang, H.; Neher, D.; Ade, H.; Bazan, G. C.; Nguyen, T.-Q. Adv. Energy Mater. 2017, 8, 1701073.

(36) Müller-Buschbaum, P. Adv. Mater. 2014, 26, 7692.

(37) Rivnay, J.; Mannsfeld, S. C.; Miller, C. E.; Salleo, A.; Toney, M. F. Chem. Rev. 2012, 112, 5488.

(38) Zhang, M.; Zhu, L.; Qiu, C.; Zhang, Y.; Liu, F. Acta. Polym. Sin. 2019, $50,352$.

(39) Ma, W.; Tumbleston, J. R.; Wang, M.; Gann, E.; Huang, F.; Ade, H. Adv. Energy Mater. 2013, 3, 864. 